\title{
Circular Polarization Plane Array Antenna composed of Truncated Feed-and Reactance-Elements using Glass-epoxy Substrates
}

\author{
YUMI TAKIZAWA \\ Institute of Statistical Mathematics \\ Research Organization of Information and Systems \\ Tokyo, Japan \\ CAHYA EDI SANTOSA \\ Center for Environmental Remote Sensing \\ Chiba University, Chiba, Japan
}

\author{
ATSUSHI FUKASAWA \\ Former Professor, Chiba University, and \\ Technical Adviser \\ Musasino Co. Ltd, Tokyo, Japan
}

\author{
JOSAPHAT TETUKO SRI SUMANTYO \\ Center for Environmental Remote Sensing \\ Chiba University, Chiba, Japan
}

\begin{abstract}
This paper presents a circular polarization plane antenna using linearly truncated triplate stripline resonator antenna. Glass-epoxy substrate is used in place of Teflon substrate for low cost fabrication. In spite the electrical dimension differs only $\sqrt{ } 2$ (root two), the dimensions of circular disk, its truncation, and feeding lines must be chosen precisely and accurately to get better characteristics of gain $12 \mathrm{~dB}$ compared to $10 \mathrm{~dB}$ by elliptic resonator with 4-antenna array.
\end{abstract}

Key-Words: - X-band circular polarization antenna, compact plane antenna, high permittivity dielectric material, axial ratio

Received: August 14, 2020. Revised: December 31, 2020. Accepted: January 16, 2021. Published: January 31, 2021.

\section{Introduction}

The authors have been attracted in microwave circular polarization plane antennas. The characteristics of wideband axial ratio and high directive gain were realized by the design using Teflon multilayer substrates.

It was found however that the conventional design brings extremely high production cost. Metalizing on the dielectric material of Teflon are not easy. And thermal pressure contact requires multiple steps for adhesive contact among different substrates of feed- and reactance-elements, and ground plate.

A novel design is given in this paper GlassReinforced Epoxy laminate (or glass-epoxy) substrates are introduced into practical design. This material is popular, low cost with enough reliability. Teflon substrates requires multiple processes to implement triplate stripline antenna and feeding routing wires.

This paper proposes a novel design of a plane antenna for circular polarization based on the linearly truncated circular disc of a feed element. It is pointed that the diameter and the truncated length of circular disc are defined to the lower and the higher frequency band edges to meet the generation conditions.

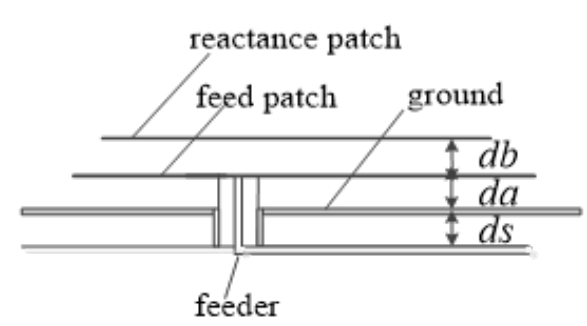

Fig. 1 Cross-sectional view of triplate stripline resonator antenna.

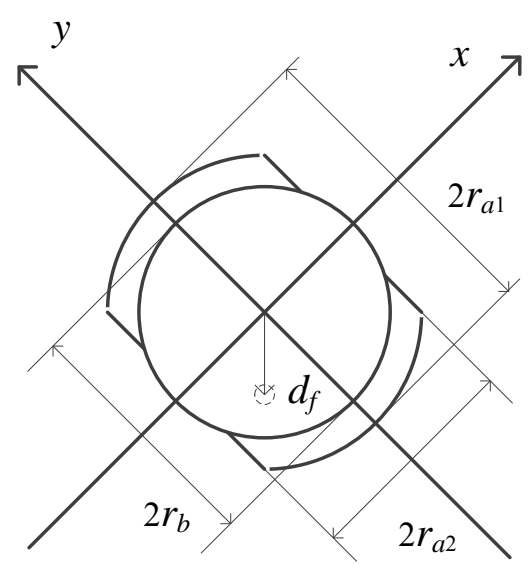

Fig.2 Feed- and reactance-elements. Ground plate and grounded collar are abbreviated. 


\section{Single Antenna}

A novel triplate stripline resonator antenna was brought by the former technologies [1-3]. The configuration of the unit antenna is shown in Fig. 1 and 2, which have been developed by Y. Takizawa and A. Fukasawa[4-11].

Figure 1 gives cross-sectional view of a stripline resonator antenna composed of feed- and reactanceelements $a$ and $b$, and the ground plate $g$. The substrate $s$ under the ground plate provides feeding routing wire for the antenna. Feed element $a$ is fed with vertical probe through substrate $s$ under the ground plate.

Figure 2 shows the top view shows linearly truncated circular feed-element and circular reactance-element. 2ra1, 2ra2 are long and short axes of feed-element, which yields $x$ and $y$ axiscomponents for a rotating polarization vector.

\section{Four Antenna Array}

\subsection{Configuration of the proposed array antenna}

An array antenna is shown in Fig. 3. Four unit antennas ai, $(i=1 \sim 4)$ are set at each quadrant around the center $O$ in $X-Y$ plane. $Z$ axis is perpendicular against $X-Y$ plane.

Each unit antenna generates right-handed polarized wave. To get right-handed polarized wave totally, each antenna must be fed by the signal with 90 degree phase delay along the left-handed circulation. $d_{f X}$ and $d_{f Y}$ shows the position of feeding point at each unit antenna.

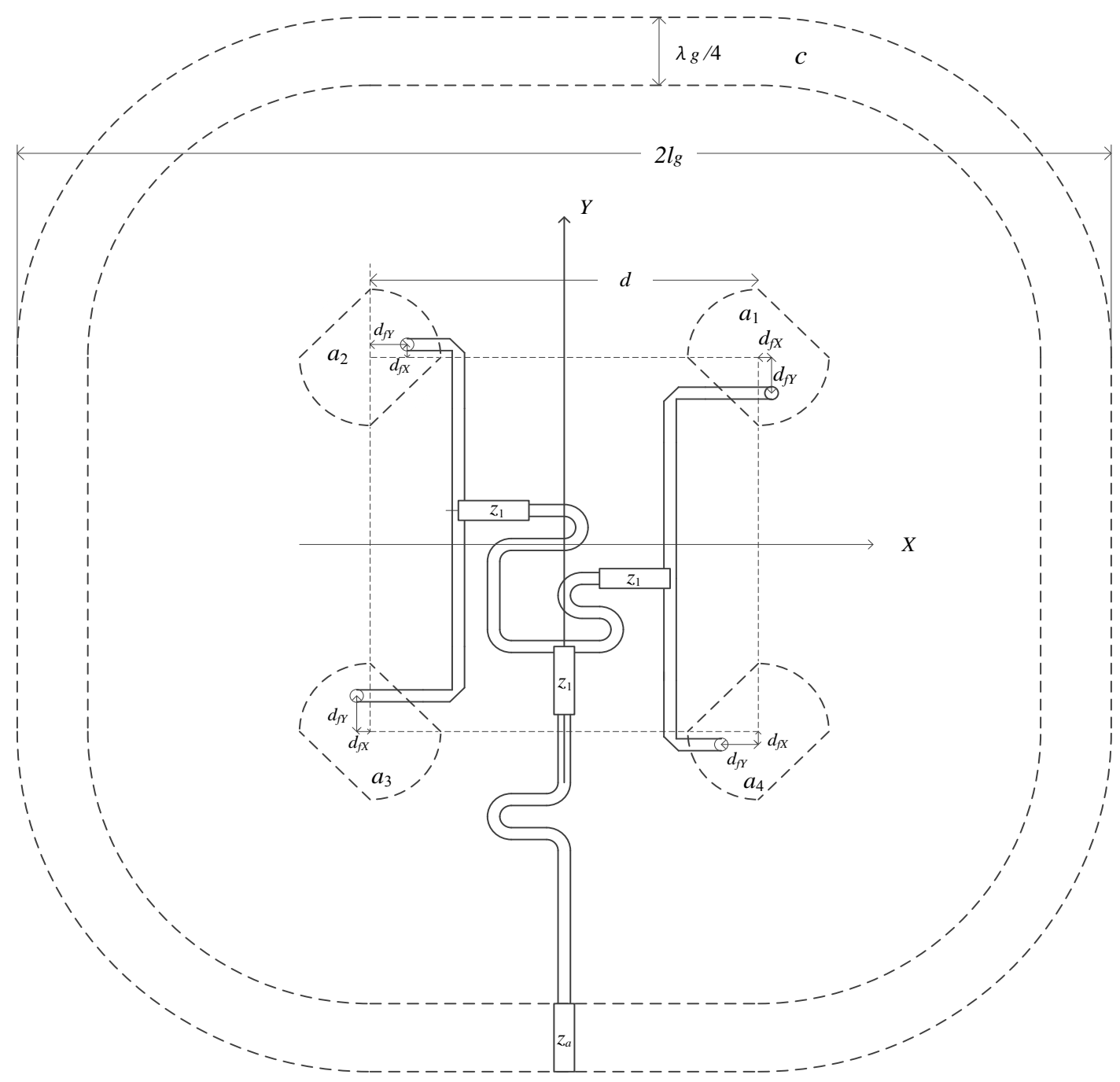

Fig. 3 Configuration of plane array antenna.

Reactance elements and dielectric substrates are abbreviated. 
The length of the ground plate $2 \lg$ must be large enough compared to the size of total space of inner conductors.

\subsection{Routing wire configuration}

This scheme forms a $\mathrm{p}$ arallel feeding for the orthogonal arrangement of four antennas. This novel configuration shown in Fig. 3 is developed by Y. Takizawa and A.Fukasawa.

The routing wires are composed with smoothed S-curves, which reduce microwave reflections observed in right angle bending, T-branches, and the other transmission line with rapid bending.

The condition of 90 degree phase difference are given between right hand elements $a 1$ vs $a 4$, and the left hand elements $a 3$ vs $a 2$. At the connection of the right and the left elements, 180 degree and 90 degree phase delay are provided by corresponding line lengths.

The dotted line $c$ in Fig. 3 shows the collar of this array with $\lambda \mathrm{g} / 4$ line and short termination for suppression of horizontal radiation. This structure has been proposed by Y. Takizawa and A. Fukasawa[6-11].

\section{Characteristics}

\subsection{Design parameters}

The central frequency and the bandwidth are designed for the X-band. The array antenna is composed of 4 unit antennas.

Frequency band;

central frequency $f_{0}=10 \mathrm{GHz}$

Dimension of the element $a$;

length along $y$ axis $\quad 2 r_{a 1}=6.6(\mathrm{~mm})$

cutting width $\quad 2 r_{a c}=1.8(\mathrm{~mm})$

length along $x$ axis $\quad 2 r_{a 2}=2\left(r_{a 1-}-r_{a c}\right)=4.8(\mathrm{~mm})$

feeding position $\quad d f X=0(\mathrm{~mm})$,

$d f Y=1.7(\mathrm{~mm})$

Dimension of the element $b$;

$$
\text { diameter } 2 r_{b}=5.4(\mathrm{~mm})
$$

Dimension of the ground element $g$ : diameter

$$
2 l_{g}=62.0(\mathrm{~mm})
$$

Relative permittivity

$$
\varepsilon r=4.6
$$

Dielectric tangent

$$
\tan \delta=0.010
$$

Thickness of metal $\quad d_{M}=0.035(\mathrm{~mm})$
Distance between $a$ and $b$ elements

Distance between $g$ and $a$ elements

Distance between $s$ and $g$

$$
\begin{aligned}
d_{a} & =1.2(\mathrm{~mm}) \\
d_{b} & =1.2(\mathrm{~mm}) \\
d s & =0.4(\mathrm{~mm})
\end{aligned}
$$

\subsection{Characteristics of the 4-Antenna Array}

The evaluation was given by $3 \mathrm{D}$ computer simulation CST in the environment constructed by C.E. Santosa, Chiba University. Return loss, input impedance, axial ratio, and directive gain are shown in Fig. 4, 5, 6, and 7. Wideband axial ratio and high directive gain were confirmed by this configuration and design.

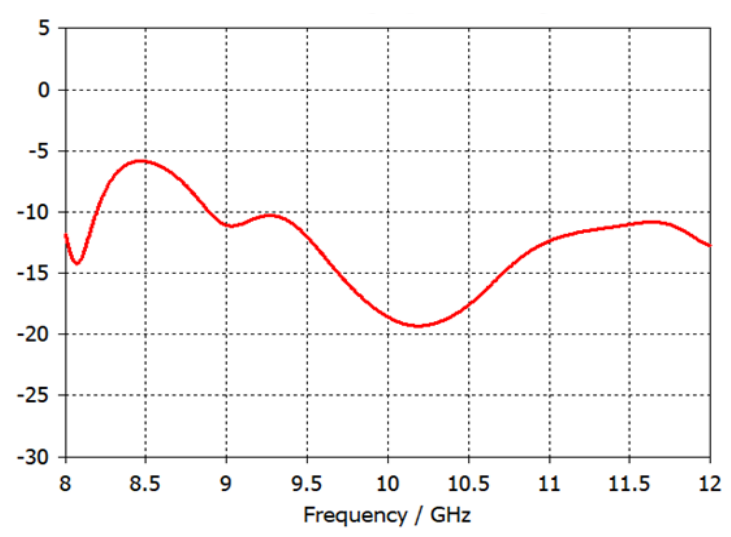

Fig. 4 Return loss.

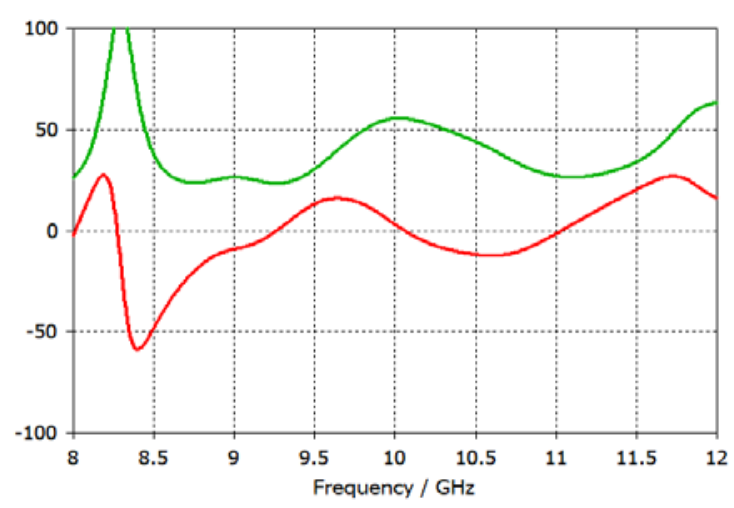

Fig. 5 Input impedance.

Green line: real part.

Red line: imaginary part 


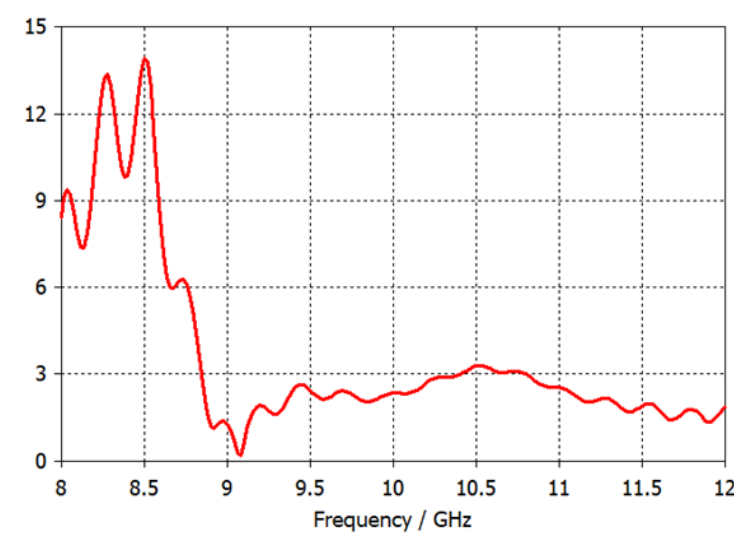

Fig. 6 Axial ratio.

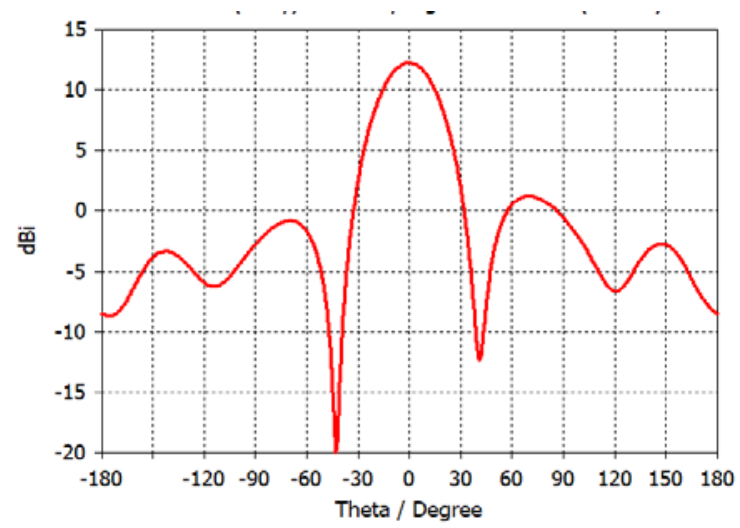

Fig. 7 Farfield gain $\left(\varphi=90^{\circ}\right)$.

\section{Conclusion}

This paper presented the results of development on a design of $\mathrm{X}$-band circular polarization plane antenna. The structure of triplate stripline resonators was composed of circular disc with linear truncation for the feed-element. Simple circular disc with different diameters was used reactance-element.

Wideband axial ratio is $20 \%$ or more of the central frequency. Higher directive gain of $12 \mathrm{~dB}$ was realized. The triplate stripline configuration with linearly truncated circular disc exceeds the directive gain compared to elliptic resonators and conventional design using square and circular disc resonators[12].

It is pointed that the structure of stripline resonator for circular polarization should be regarded about configuration of resonator itself.

\section{Acknowledgment}

This work was supported by MEXT/JSPS KAKENHI Grant Number 17K00067, and the scholarship donations given by Musasino Co. Ltd, and Joint Research 2019-ISMCRP-2023.

The authors express their sincere gratitude for effective supports and advices by the DirectorGeneral. Prof. H. Tsubaki,, ISM and Prof. N. Kashiwagi, ISM.

And the authors express their sincere gratitude for kind supports by Mr. M. Abe, CEO, and Mr. M. Kise, General manager of R\&D, Musasino Co.Ltd.

\section{References:}

[1] Yagi S., Mushiake Y., Yagi-Uda Antenna, Sasaki Co., 1954.

[2] Haneishi M., et al, Analysis, design, and measurement of small and low-profile antenna, Artech House (U.S.A), pp.1-270, 1991.

[3] Haneishi M., et al, Radiation properties of ringshaped microstrip antenna array, IEICE, Trans. on Communications, vol. E78-C, pp.995-1001, 1995.

[4] Takizawa Y., Fukasawa A., Microwave Patch Antenna with Circular Polarization for Environmental Measurement, Journal of Electromagnetics, vol. 2, pp.1-6, June 27, 2017.

[5] Takizawa Y., Fukasawa A., Novel Structure and the Characteristics of a $\mathrm{M}$ icrowave Circular Polarization Antenna, WSEAS 
Transaction on Communications, vol. 16, pp. 184-191, 2017.

[6] Takizawa Y., Fukasawa A., Circular Polarization Array Antenna with Orthogonal Arrangement and Parallel Feeding by Smoothed Routing Wires, Journal of Electromagnetics, Vol. 3, pp. 14-19, Apr. 11, 2018.

[7] Fukasawa A., Takizawa Y., Circular Polarization Array Antenna with Orthogonal Arrangement and Parallel Feeding by Simplified Routing Wires, Journal of Electromagnetics, Vol. 3, pp. 3 -8, Apr. 11, 2018.

[8] Takizawa Y., Fukasawa A., 16-Antenna Array for Circular Polarization with Wideband Axial Ratio and Enhanced Directivity, Journal of Electromagnetics, Vol. 3, pp. 20-26, Oct. 26, 2018.

[9] Takizawa Y., Fukasawa A., Circular Polarization Plane Antenna Array by AntiParallel Arrangement with Simplified Routing Wire, Journal of Electromagnetics, Vol. 3, pp. 27-32, Oct. 26, 2018.

[10] Takizawa Y., Fukasawa A., 64-Antenna Array for Circular Polarization with Smoothed Routing Wires and Grounded Square Collar," Trans. on Communications, vol. 18, pp. 49-56, 2019.

[11] Fukasawa A., Takizawa Y., Santosa C.E., Josaphat T.S Sumantyo, Circular Polarization Antenna with Truncated Feed-and ReactanceElements using GlassEpoxy Substrates, International Journal of Communications, vol. 4, 23-27, 2019.

\section{Creative Commons Attribution License 4.0 (Attribution 4.0 International, CC BY 4.0)}

This article is published under the terms of the Creative Commons Attribution License 4.0

https://creativecommons.org/licenses/by/4.0/deed.en_US 